\title{
Realignment Course in Mathematics: design of an online valuable experience for students
}

\author{
Barana, Alice; Bogino, Alessandro; Fioravera, Michele; Floris, Francesco; Marchisio, \\ Marina
}

Department of Mathematics, University of Turin, Italy.

\begin{abstract}
Supporting students in the transition from secondary school to university is an important action to be taken in order to increase the number of people with tertiary education degrees; it has positive consequences on the citizens' working perspective and on the national economic growth. To this purpose, in the academic year 2014/2015 the University of Turin started the Project Orient@mente, a platform of self-paced open online courses aimed to guide students in the choice of a scientific university program. This paper focuses on the design of the Realignment Course in Mathematics, provided by the platform and aimed at the self-paced review and integration of knowledge that students are required to acquire during secondary school. Materials are created and structured according to the User Experience Design for Learning, with the aim of providing a valuable learning experience to students. Contents are delivered through an accessible learning management system integrated with an advanced computing environment and an automatic assessment system. Data from the usage of the activities and from the results of the online tests are analyzed in order to validate the design choices and the coherent structure of the course.
\end{abstract}

Keywords: Automatic Assessment; Design for Learning; E-Learning; Mathematics Education; Realignment Course; Self-paced open online courses. 


\section{Introduction}

For all European countries which subscribed Europe 2020 agreements, increasing the number of people who completed tertiary education should be a major goal finalized to improve the citizens' working perspectives and the national economic growth (European Commission, 2015). In Italy, the number of enrolments to universities has registered a growing trend for the last five years, even if less than $30 \%$ of students graduates on time and the same amount abandons University before graduating. STEM subjects (Science, Technology, Engineering and Mathematics) are the most attractive, earning 36\% of enrolments in the last academic year, a growing percentage for the last 4 years; nevertheless, they register the highest number of abandons - beyond 35\% - and the lowest rate of on-time graduations (less than 15\%) (ANVUR, 2016) (MIUR, 2017).

In the light of these data, it is clear that tertiary education needs actions for orienting students in the choice of an appropriate university area and for guiding them through the first years, especially in scientific courses where the "hard subjects" such as Mathematics or Physics are prevalent in the curricula. Many universities, in Italy as well as around the world, have chosen blended or e-learning modalities to deliver orienting or refresh courses, in order to actively engage a wider number of students (Calidoni, et al., 2009) (Urbonaite, et al., 2013). In this scenery the Project Orient@mente started at the University of Turin (Italy) in the academic year 2014/15 and it is currently underway. The Project consists in the development of a platform for the fruition of self-paced open online courses (http://orientamente.unito.it/). It is aimed to support three main valuable actions for students who intend to apply to a scientific program at the university: guidance to the university offer, automated testing of basic concepts and competences, self-paced review and integration of knowledge acquired during secondary school (Barana, et al., 2017). In line with the three purposes, three different kinds of self-paced open online courses have been developed:

- $\quad$ orienting courses with information about the study courses and future careers;

- testing courses to verify students' basic knowledge and skills and to enhance their awareness about their initial situation;

- realignment courses to strengthen their competences and fill the gaps in their preparation.

The online courses are structured according to educational models grown thanks to the experience and the research in e-learning and digital asset carried out by the University, especially in the use of a learning management system integrated with an advanced computing environment and an automated assessment system (Brancaccio, et al., 2015)

This paper focuses on the design of the Realignment Courses (RC), which pay great attention to providing users a valuable learning experience. The $\mathrm{RC}$ in Mathematics is 
analyzed in the light of models of learning design from the literature; the discussion is supported by data from the activities usage.

\section{State of the art}

Technologies and virtual learning environments can facilitate good learning experiences through systems for computer mediated communication and collaboration, cognitive tools for knowledge representation and construction, environments for problem solving, simulation and case-based learning (Jonassen, et al., 1995). In particular, the structure of learning environments, the possibilities of interaction, immediate feedback and assessment promote the development of metacognition and self-regulation, which are at the core of learning processes (Persico \& Steffens, 2017).

In the design of web-based learning activities, it is important to place the learner at the center of the learning process. Thus, models based on the user experience achieve a great effectiveness in the delivering of learning. The University of Waterloo has developed the "User Experience Design for Learning Honeycomb" (UXDL Honeycomb), a design framework for creating online courses which relies on theories of information architecture and user experience (University of Waterloo). According to the UXDL Honeycomb, in order to provide valuable and interesting learning experiences, contents should be:

- useful for the learning process. In particular, they should help the learner avoid cognitive overload, select, organize, and integrate information, process new materials and construct a schema;

- desirable: in order to create a positive approach to learning, which positively influences motivation, materials should be visually appealing and they should promote reflection;

- accessible, to accustomize to everyone's needs;

- credible, which relates to the quality: respect for academic integrity, consistency of the activities with the outcomes, professional appearance, barrier-free learning;

- intuitive: materials should be easy to find when navigating the course, and easy to use or self-explanatory.

\section{The Realignment Courses}

Orient@mente's Realignment Courses consist of four self-paced open online courses respectively on Biology, Chemistry, Physics and Mathematics. They can be addressed to three main different aims: helping students attend the lessons of the first year of university courses, helping students pass their first university exams and reducing the dropout rate of the first year of university. Students can attend them to review, reinforce and integrate their basic knowledge on the discipline, to fill the gaps in their initial preparations and to prepare for the admission tests. 
The design of the RCs has been conducted according to the strategies pointed out by the User Experience Design for Learning model. The design of the four RCs followed similar methods; however, disciplinary needs led to slightly different choices of tools and materials, therefore the following analysis will focus on the RC in Mathematics.

The whole structure of the RCs is conceived to make them useful: in fact, the courses are highly structured, composed of a list of modules in turn split into smaller submodules which focus on specific topics. The purpose is to break contents into shorter and studentcontrolled chunks instead of giving a long and continuous presentation of material; thus, students are facilitated in selecting, organizing, and integrating new materials with relevant prior knowledge. In addition, explorative and interactive materials fill the courses in order to foster generative processing and help students actively construct new concepts.

The experience of navigating through the online courses wants to be intuitive for learners with the goal to make content and activities easy to find and easy to use. The design of the interface and the underlying technology are invisible.

To create valuable online courses for every type of learner, accessibility is provided first of all by the course design and it's checked periodically with an online validator and monitor, who checks whether materials are aligned to the official guidelines for accessibility of webpages (MIUR, 2013). Moreover, the default platform aspect uses the high-legibility font EasyReading ${ }^{\circ}$, which maximizes the website legibility by simplifying the reading for dyslexic students, and looking good for everyone. To the purpose of making its structure clear and of setting shared expectations between students and instructors, at the top of each $\mathrm{RC}$ there are a general description of the learning outcomes and a mind map of the topics covered. Similar descriptions are also inserted within modules and submodules. To facilitate the learning process (and to adapt to the widest range of learning styles), each topic is presented in different modalities, such as texts, video lessons, tests and interactive files. More specifically, lessons are organized according to a regular pattern consisting of the following activities:

- Theory - usually printable or online readable books, made with Moodle books;

- Explore - interactive activities of exploration or simulation, created with Maple, an Advanced Computing Environment integrated within Moodle, which allows several types of representation, computation, simulation and interaction;

- Applications - Moodle pages with theory applications and curiosities;

- Quizzes - automatically assessed online tests made through Maple TA, a system particularly suitable for STEM. It supports open mathematical answers graded independently of their form, algorithm-based variables and immediate feedback;

- Exercises with their solutions - downloadable and readable online sheets. 
At the end of every module there is a final test on the whole module's theory (Figure1). All these aspects of careful organization and design are fundamental to gain learners' trust and make courses credible. The quality of materials and activities is also guaranteed by the whole process of design of the courses. The process was based on team-working and modeled on the Deming cycle: plan-do-check-act (Walasek, et al., 2011). Preliminary studies and planning with managers and digital experts from the University have been carried out to define suitable instruments and methodologies. Contents were created in collaboration with professors. The platform developers continuously adjust the materials and the services offered according to feedback collected from users through specific and ever-open surveys.

Lastly, the courses' structure and appearance make them desirable. The order and clarity of the pages contribute to their appealing look and to offer a visual image of the learning path to cover; activities and tests foster meta-reflection and self-regulation, which are key points to promote motivation and the achievement of learning outcomes. In fact, with online tests, students can work independently and whenever they want: immediate automatic feedback allows them to acknowledge their level of preparation. Algorithm-based questions with multiple attempts allow students to repeat reasoning, not results, until mastered, additionally offering many chances of drills for the admission tests (Barana \& Marchisio, 2016).

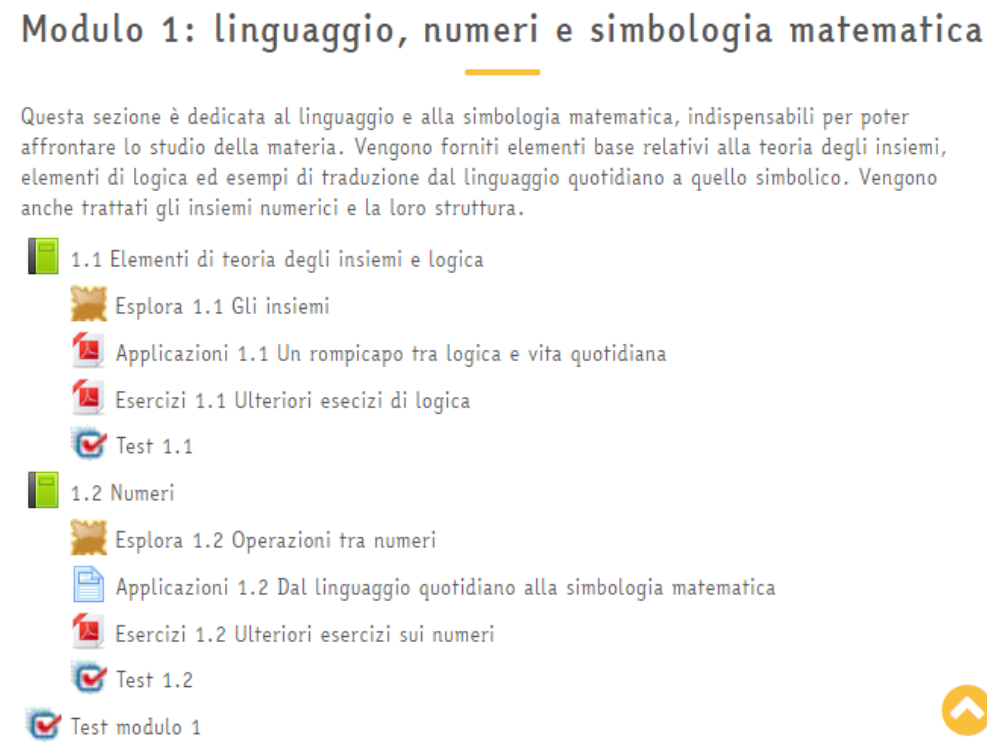

Figure 1. Structure of a module. Source: Orient@mente platform (2018). 


\section{Analysis of the Realignment Course in Mathematics}

Since its opening on July $14^{\text {th }}, 2015$, Orient@mente registrations have been following a regularly increasing trend, reaching 10135 users. Similarly, enrollments to the RC in Mathematics have been growing to reach 1052 users since its opening on, 2015 October $20^{\text {th }}, 2015$ (data are updated to January $15^{\text {th }}, 2018$ ). The materials of the RC are frequently visited by users: the total number of logs (accesses) to the course and to the sections amounts to 61134; the logs to the resources of the RC of Mathematics are reported in Table 1 .

Table 1. Resources and activities logs

\begin{tabular}{ccc}
\hline Resource or activity & $\begin{array}{c}\text { Logs (absolute } \\
\text { frequency) }\end{array}$ & $\begin{array}{c}\text { Logs average } \\
\text { (daily) }\end{array}$ \\
\hline Book & 101462 & 110.77 \\
Maple Worksheet & 3099 & 3.38 \\
Page & 3021 & 3.30 \\
MapleTA quiz & 5881 & 6.42 \\
PDF Files & 10705 & 11.69 \\
\hline Source: Report from Orient@mente's platform (2018)
\end{tabular}

Source: Report from Orient@mente’s platform (2018)

In order to evaluate the effectiveness of the design of the activities, their coherence with the modules' learning objectives and their consistency with the assessment, an analysis on the results of the online tests has been conducted. We considered all the results to the tests inserted at the end of each submodule and module, in a scale from 0 to 10 , selecting the best result for each student in case of repeated attempts (users have unlimited attempts for the tests). For each result, we have considered whether the user had completed the other activities or used the other resources in the same module before attempting the test. We obtained two groups: Group 1 is composed of the results of users who have carried out the test without taking advantage of the resources and activities in the RC; Group 2 is composed of learners presenting log-data from the activities and resources of the module. In the 9 modules there are 27 tests, each one attempted by a number of users which ranges from a minimum of 46 to a maximum of 462 (average: 150). The sample size considered is 4052; 518 belong to Group 1 and 3534 to Group 2. Table 2 shows the average results to the tests of the two groups: it is clear that students who used the activities before the test did better than those who completed the test just relying on their own knowledge. The statistical significance of the difference between the two groups is confirmed by an analysis of covariance (ANCOVA) carried out on the results of the two groups, using the means of the results of the tests as covariate variable $(\mathrm{p}=0.007$, squared eta $=0.1)$. 


\section{Synthesis of the results and discussion}

The statistics shown in the previous section highlight that the activities in the RC of Mathematics are frequently used by students; moreover, they are inserted within a welldesigned learning path, they are consistent with learning outcomes and assessment and they help students in the revision of the contents. Emphasis to the relation between learning outcomes and assessment is given to further motivate students and to help them understand the importance of the resources and activities of the course. Therefore, the RCs can be a valid tool for students transitioning from high school to university, allowing them to reinforce their competence. The continually increasing number of enrolments and usage of the course confirms that it is employed by students. Similar results are also achieved in the other three Realignment Courses, even though their analysis is beyond the purposes of this paper. Moreover, the interactive activities and online tests allow some good practices, that are to assess the achievement of learning outcomes throughout the learning experience and to make assessments become a part of the instructional process. Thus, the RC can pursue several goals that are not strictly related to disciplinary contents, such as the enhancement of the ability to self-evaluate and self-regulate one's learning and the development of digital competences.

Table 2. Results of the tests of the $\mathrm{RC}$ in Mathematics for the two groups of users

\begin{tabular}{cccc}
\hline Groups & Frequency & Average result & Standard deviation \\
\hline 1 & 518 & 6.67 & 2.40 \\
2 & 3534 & 7.07 & 2.56 \\
\hline Total & $\mathbf{4 0 5 2}$ & $\mathbf{6 . 9 6}$ & $\mathbf{2 . 4 3}$ \\
\hline
\end{tabular}

\section{Conclusion}

Orient@mente is not simply a static archive of materials. Challenged to actively try and explore, students can become more aware of their attitude, knowledge and skills, and face with more self-confidence the transition from high school to University. The clear structure of RCs promotes self-organization and everyone is left free to make use of its contents according to their needs. It also has a positive influence on time management, which is key factor not only to succeed at university, but also to succeed at work. The courses can be enjoyed not only by students who are going to enroll to the University of Turin, but by everyone who wants to start a scientific program in any university, since the disciplinary requirements and transversal competence needed are similar everywhere. 
In the past three years, Orient@mente has opened many possibilities of research in several directions: strengthening connections with other social university e-learning environments, studying the role of automatic assessment in improving learning, extending similar opportunities to other disciplines. The research is still ongoing; for example, some materials of the RC of Mathematics are going to be renewed by using innovative tools to deliver the theoretical contents, such as slideshow systems that allow to insert questions and interactive activities in the theory section, embedding them at appropriate moments: students can grasp key concepts before moving on. As a consequence, effectiveness of the quizzes and interactive files will be further maximized.

\section{References}

ANVUR (2016). Rapporto biennale sullo stato del sistema universitario e della ricerca.

Barana, A., \& Marchisio, M. (2016). Ten good reasons to adopt an automated formative assessment model for learning and teaching Mathematics and scientific disciplines. Procedia - Social and Behavioral Sciences, 228, 608-613.

Barana, A., Bogino, A., Fioravera, M., Marchisio, M., \& Rabellino, S. (2017). Open platform of self-paced moocs for the continual improvement of academic guidance and knowledge strenghtening in tertiary education. JE-LKS. Journal of E-Learning and Knowledge Society, 13(3), 109-119.

Brancaccio, A., Demartini, C., Marchisio, M., Palumbo, C., Pardini, C., Patrucco, A., \& Zich, R. (2015). Problem Posing and Solving: Strategic Italian Key Action to Enhance Teaching and Learning of Mathematics and Informatics in High School. Proceedings International Computer Software and Applications Conference (COMPSAC 2015), Volume 2, 845-850.

Calidoni, P., Gola, E., Isu, G. C., \& Satta, R. (2009). Orientamento e riallineamento universitario on-line: progettazione e prova di servizi nel progetto UniSOFIA. Italian Journal of Educational Research (2-3), 19-26.

European Commission (2015). EUROPE 2020 - A strategy for smart, sustainable and inclusive growth. Europe 2020: an Overview, Annex 1.

Jonassen, D., Davidson, M., Collins, M., Campbell, J., \& Haag, B. B. (1995). Constructivism and Computer-Mediated Communication in Distance Education. American Journal of Distance Education, 9(2), 7-26.

MIUR (2013). Decreto 20 marzo 2013: Modifiche all'allegato A del decreto 8 luglio 2005 del Ministro per l'innovazione e le tecnologie, recante: «Requisiti tecnici e i diversi livelli per l'accessibilita' agli strumenti informatici». (Prot. 195/Ric). (13A07492).

MIUR (2017). Focus “Gli immatricolati nell'a.a. 2016/2017 il passaggio dalla scuola all'università dei diplomati nel 2016”.

Persico, D., \& Steffens, K. (2017). Self-Regulated Learning in Technology Enhanced Learning Environments. In E. Duval, M. Sharples \& R. Sutherland (Eds.), Technology Enhanced Learning (115-124). Springer. 
Urbonaite, V., Winkler, S., \& Körner, A. (2013). Various Usage of Maple T.A. in Mathematics, Modelling and Simulation. ERK - International Electrotechnical and Computer Science Conference, 173-176. Portoroz.

Walasek, T. A., Kucharczyk, Z., \& Morawska-Walasek, D. (2011). Assuring quality of an e-learning project through the PDCA approach. Archives of Materials Science and Engeneering, 48(1), 56-61.

University of Waterloo, Center of Extended Learning (CEL). User Experience Design for Learning. Retrived from http://cel.uwaterloo.ca/honeycomb/index.html 